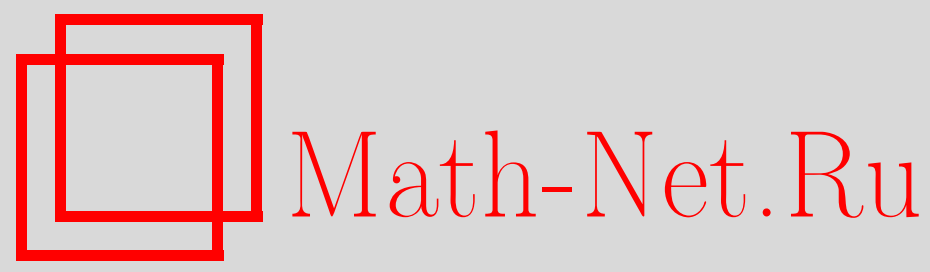

Ю. С. Харин, Е. В. Вечерко, Статистическое оценивание параметров модели вкраплений в двоичную цепь Маркова, Дискрет. матем., 2013, том 25, выпуск 2, 135-148

DOI: https://doi.org/10.4213/dm1241

Использование Общероссийского математического портала Math-Net.Ru подразумевает, что вы прочитали и согласны с пользовательским соглашением http://www.mathnet.ru/rus/agreement

Параметры загрузки:

IP: 54.162 .127 .20

26 апреля 2023 г., 13:19:15 


\title{
Статистическое оценивание параметров модели вкраплений в двоичную цепь Маркова
}

(c) 2013 г. Ю. С. Харин, Е. В. Вечерко

\begin{abstract}
В статье рассматриваются математические модели вкраплений в двоичную цепь Маркова, возникающие в задачах стеганографии. Построены и исследованы статистические оценки модельных параметров, основанные на частотных статистиках и выборочных корреляциях. Разработан полиномиальный алгоритм вычисления функции правдоподобия, на основе которого построены оценки максимального правдоподобия. Представлены результаты компьютерных экспериментов.
\end{abstract}

\section{1. Введение}

В настоящее время стеганография стремительно развивается и используется при решении задач защиты информации $[1,4,3,6,5,2,7,8]$. Несмотря на большое число публикаций, в литературе недостаточно внимания уделено теоретическим аспектам стеганографической защиты информации. Вероятностно-статистические аспекты стеганографии мало изучены, поэтому проблема построения и анализа математических моделей вкраплений (встраивания сообщений в контейнеры) актуальна $[7,9]$.

В качестве математических моделей контейнеров в стеганографии могут использоваться двоичные случайные последовательности и случайные поля. Использование схемы независимых испытаний Бернулли в качестве модели контейнера позволяет построить статистические критерии обнаружения вкраплений [7] и теоретически оценить их мощности. Однако эта модель не учитывает существующих зависимостей в реальных статистических данных, используемых в качестве контейнеров. В данной статье используется обобщение модели из [7] - марковская модель зависимости.

Целью данной статьи является исследование вероятностных свойств и статистическое оценивание параметров для моделей двоичной цепи Маркова с вкраплениями.

Статья имеет следующую структуру. В разделе 2 дается обоснование марковской модели вкраплений и двух ее частных случаев: классической модели побитового вкрапления и новой модели q-блочного вкрапления. Раздел 3 содержит результаты по вероятностным распределениям $s$-грамм и статистическим оценкам параметров на основе этих распределений для модели побитового вкрапления. Раздел 4 посвящен оценкам максимального правдоподобия параметров для модели побитового вкрапления, а раздел 5 - для модели блочного вкрапления. В разделе 6 теоретические результаты иллюстрируются результатами компьютерных экспериментов. 


\section{2. Математические модели цепи Маркова с вкрап- лениями}

Введем обозначения: $V=\{0,1\}$ - двоичный алфавит, $V_{T}-$ пространство двоичных $T$-мерных векторов, $\mathbb{N}$ - множество натуральных чисел, $I\{A\}$ - индикатор события $A, u_{t_{1}}^{t_{2}}=\left(u_{t_{1}}, \ldots, u_{t_{2}}\right) \in V_{t_{2}-t_{1}+1}\left(t_{1}, t_{2} \in \mathbb{N}, t_{1} \leqslant t_{2}\right)$ - двоичная строка из $t_{2}-t_{1}+1$ символов, $w(\cdot)$ - вес Хемминга, $\mathfrak{L}\{\xi\}$ - закон распределения вероятностей случайной величины $\xi, \mathfrak{B}(\theta)$ - закон распределения вероятностей Бернулли с параметром $\theta \in$ $[0,1]: \mathbf{P}\{\xi=1\}=1-\mathbf{P}\{\xi=0\}=\theta$.

Будем предполагать, что контейнер для встраивания сообщения есть двоичная последовательность $x_{1}^{T}=\left(x_{1}, x_{2}, \ldots, x_{T}\right) \in V_{T}, x_{t} \in V, t=1, \ldots, T$, являющаяся однородной двоичной цепью Маркова 1-го порядка с матрицей вероятностей одношаговых переходов $P=\left(p_{v_{0}, v_{1}}\right), v_{0}, v_{1} \in V$ :

$$
\begin{gathered}
P=P(\varepsilon)=\frac{1}{2}\left(\begin{array}{cc}
1+\varepsilon & 1-\varepsilon \\
1-\varepsilon & 1+\varepsilon
\end{array}\right), \\
p_{v_{0}, v_{1}}=\mathbf{P}\left\{x_{t+1}=v_{1} \mid x_{t}=v_{0}\right\}=\frac{1}{2}\left(1+(-1)^{v_{0}+v_{1}} \varepsilon\right),|\varepsilon|<1 .
\end{gathered}
$$

Здесь $\varepsilon-$ параметр модели: случай $\varepsilon=0$ соответствует схеме независимых испытаний и исследован в [7]; случай $\varepsilon>0$ учитывает зависимость типа притяжения, $\varepsilon<0$ - зависимость типа отталкивания. Отметим, что цепь Маркова (2) удовлетворяет условиям эргодичности [10] и имеет равномерное стационарное распределение вероятностей $\pi=(1 / 2,1 / 2)$. Далее будем полагать, что цепь Маркова (2) является стационарной, то есть ее начальное распределение совпадает с $\pi$.

Отметим, что представленная модель контейнера достаточно хорошо согласуется с реальными статистическими данными. Например, при анализе $10^{2}$ случайно выбранных $512 \times 512$ изображений в градациях серого из базы данных [8] установлено, что $\left|\mathbf{P}\left\{x_{t}=1\right\}-\frac{1}{2}\right| \leqslant 0.0021$, а оценка для параметра $\varepsilon$ равна 0.07 .

Обычно до встраивания в контейнер сообщение подвергается криптографическому преобразованию [11], устраняющему статистическую избыточность, поэтому далее полагаем, что сообщение $\xi_{1}^{M}=\left(\xi_{1}, \ldots, \xi_{M}\right) \in V_{M}, M \leqslant T$, является последовательностью $M$ независимых случайных величин Бернулли:

$$
\mathfrak{L}\left\{\xi_{t}\right\}=\mathfrak{B}\left(\theta_{1}\right), \mathbf{P}\left\{\xi_{t}=j\right\}=\theta_{j}, j \in V, \theta_{1}=1-\theta_{0}, t=1, \ldots, M
$$

На практике, как правило (11]), $\left\{\xi_{t}\right\}$ имеет симметричное распределение вероятностей: $\theta_{1}=\theta_{0}=1 / 2$.

Стегоключ $\gamma_{1}^{T}=\left(\gamma_{1}, \ldots, \gamma_{T}\right) \in V_{T}$, определяет моменты времени, в которые биты сообщения $\xi_{1}^{M}$ вкрапляются в последовательность $x_{1}^{T}$. Так как $V$ - двоичный алфавит, то распространенные в стеганографии методы вкрапления информации "LSB replacement" и " \pm 1 embedding" [12] тождественны и имеют вид:

$$
Y_{t}=\gamma_{t} \xi_{\tau_{t}}+\left(1-\gamma_{t}\right) x_{t}= \begin{cases}x_{t}, & \text { если } \gamma_{t}=0 \\ \xi_{\tau_{t}}, & \text { если } \gamma_{t}=1\end{cases}
$$

где $Y_{1}^{T}=\left(Y_{1}, \ldots, Y_{T}\right)$ - случайная стегопоследовательность, содержащая сообщение, $\tau_{t}=\sum_{j=1}^{t} \gamma_{j} \leqslant M$ при $\gamma_{t}=1$ определяет номер бита сообщения $\xi_{\tau_{t}}$ для вкрапления в момент времени $t$. 
Будем полагать, что стегоключ (ключевая последовательность) $\gamma_{1}^{T}=\left(\gamma_{1}, \ldots, \gamma_{T}\right)$ есть последовательность независимых случайных величин, имеющих бернуллиевский закон распределения вероятностей:

$$
\mathfrak{L}\left\{\gamma_{t}\right\}=\mathfrak{B}(\delta), \mathbf{P}\left\{\gamma_{t}=1\right\}=1-\mathbf{P}\left\{\gamma_{t}=0\right\}=\delta, t=1, \ldots, T .
$$

Введем в рассмотрение еще одну специальную $q$-блочную модель стегоключа $(q \in \mathbb{N})$ при $T=K q$, которая при $q=1$ совпадает с (4). Для этого вначале разобьем последовательность $x_{1}^{T}$ на блоки длины $q>1(T=K q, K \in \mathbb{N}): x_{(1)}=x_{1}^{q}, x_{(2)}=$ $x_{q+1}^{2 q}, \ldots, x_{(K)}=x_{(K-1) q+1}^{K q}$. Введем вспомогательные независимые случайные величины $\zeta_{k} \in V, \mathfrak{L}\left\{\zeta_{k}\right\}=\mathfrak{B}(\delta), k=1, \ldots, K$, которые отвечают за выбор блоков $\left\{x_{(k)}\right\}$ для вкрапления сообщения: если $\zeta_{k}=1$, то в один из наудачу выбранных битов блока $x_{(k)}$ вкрапляется один бит сообщения, иначе вкрапление не производится. Сразу же отметим, что для такой модели стегоключа максимальная пропускная способность стегосистемы уменьшается до $K=T / q$ бит. В дальнейшем будем рассматривать случай $q=2$ (случай $q>2$ исследуется аналогично).

При $q=2$ стегоключ $\gamma_{1}^{T}$ состоит из независимых пар:

$$
\left(\gamma_{2 k-1}, \gamma_{2 k}\right)=\left\{\begin{array}{l}
(0,0), \text { если } \zeta_{k}=0, \\
(0,1), \text { если } \zeta_{k}=1, \eta_{\tau_{k}}=0, \quad k=1, \ldots, K, \\
(1,0), \text { если } \zeta_{k}=1, \eta_{\tau_{k}}=1,
\end{array}\right.
$$

где $\left\{\eta_{k}\right\}$ - независимые двоичные случайные величины с распределением вероятностей $\mathfrak{L}\left\{\eta_{k}\right\}=\mathfrak{B}(1 / 2), \tau_{k}=\sum_{j=1}^{k} \zeta_{j}$. Отметим, что при таком построении стегоключей мощность множества всевозможных стегоключей уменьшается до $3^{T / 2}<2^{T}$. C учетом (5) для условных вероятностей $q_{t, u_{0}, u_{1}}=\mathbf{P}\left\{\gamma_{t}=u_{1} \mid \gamma_{t-1}=u_{0}\right\}, u_{0}, u_{1} \in V$, справедливы соотношения

$q_{2 k-1, u_{0}, u_{1}}=\left(\begin{array}{cc}1-\delta / 2 & \delta / 2 \\ 1-\delta / 2 & \delta / 2\end{array}\right)_{u_{0}, u_{1}}, q_{2 k, u_{0}, u_{1}}=\left(\begin{array}{cc}1-\delta(2-\delta)^{-1} & \delta(2-\delta)^{-1} \\ 1 & 0\end{array}\right)_{u_{0}, u_{1}}$.

Здесь учтено, что по построению $\gamma_{2 k-1}$ и $\gamma_{2 k-2}$ независимы.

Случайные последовательности $\left\{x_{t}\right\},\left\{\xi_{t}\right\},\left\{\gamma_{t}\right\}$ предполагаются независимыми в совокупности.

Заметим, что с практической точки зрения наибольшего внимания в рамках рассматриваемой здесь марковской модели вкрапления (2)-(5) заслуживает наиболее трудный для стегоаналитика случай $\theta_{0}=\theta_{1}=1 / 2$ в (2), так как в этом случае при вкраплении одномерное распределение вероятностей не искажается: $\mathbf{P}\left\{x_{t}=1\right\}=\mathbf{P}\left\{x_{t}=0\right\}=\mathbf{P}\left\{\xi_{t}=1\right\}=\mathbf{P}\left\{\xi_{t}=0\right\}=1 / 2, t=1,2, \ldots, T$.

\section{3. Распределения вероятностей s-грамм и основан- ные на них оценки параметров $\varepsilon, \delta$ для модели побитового вкрапления}

Рассмотрим $s$-мерные распределения стегопоследовательности $\left\{Y_{t}\right\}(s \in \mathbb{N})$ :

$$
p_{v_{0}, \ldots, v_{s-1}}^{(s)}=\mathbf{P}\left\{Y_{t}=v_{0}, \ldots, Y_{t+s-1}=v_{s-1}\right\}=\mathbf{P}\left\{Y_{t}^{t+s-1}=v_{0}^{s-1}\right\} \in V, t \in \mathbb{N} .
$$

Воспользуемся известным вспомогательным утверждением [13]. 
Лемма 1. Если $P=\frac{1}{2}\left(\begin{array}{cc}1+\varepsilon & 1-\varepsilon \\ 1-\varepsilon & 1+\varepsilon\end{array}\right),|\varepsilon| \leqslant 1$, то для любого натуралъного $k \in \mathbb{N}$ справедливо соотношение

$$
P^{k}=\frac{1}{2}\left(\begin{array}{cc}
1+\varepsilon^{k} & 1-\varepsilon^{k} \\
1-\varepsilon^{k} & 1+\varepsilon^{k}
\end{array}\right),\left(P^{k}\right)_{v_{0}, v_{1}}=\frac{1}{2}\left(1+(-1)^{v_{0}+v_{1}} \varepsilon^{k}\right), v_{0}, v_{1} \in V .
$$

Теорема 1. Если имеет место модель побитового вкрапления (2)-(4), то для $s$-мерных распределений вероятностей стегопоследовательности $\left\{Y_{t}\right\}$ nри $s \in$ $\{1,2,3\}$ справедливы формуль

$$
\begin{gathered}
p_{v_{0}}^{(1)}=(1-\delta) / 2+\delta \theta_{v_{0}} \\
p_{v_{0}, v_{1}}^{(2)}=(1-\delta)^{2} p_{v_{0}, v_{1}} / 2+\delta(1-\delta)\left(\theta_{v_{1}}+\theta_{v_{0}}\right) / 2+\delta^{2} \theta_{v_{0}} \theta_{v_{1}} \\
p_{v_{0}, v_{1}, v_{2}}^{(3)}=(1-\delta)^{3} p_{v_{0}, v_{1}} p_{v_{1}, v_{2}} / 2+\delta(1-\delta)^{2}\left(\theta_{v_{0}} p_{v_{1}, v_{2}}+\theta_{v_{1}}\left(P^{2}\right)_{v_{0}, v_{2}}+\theta_{v_{2}} p_{v_{0}, v_{1}}\right) / 2+ \\
+\delta^{2}(1-\delta)\left(\theta_{v_{0}} \theta_{v_{1}}+\theta_{v_{0}} \theta_{v_{2}}+\theta_{v_{1}} \theta_{v_{2}}\right) / 2+\delta^{3} \theta_{v_{0}} \theta_{v_{1}} \theta_{v_{2}}, v_{0}, v_{1}, v_{2} \in V
\end{gathered}
$$

Доказательство. По формуле полной вероятности с учетом (2)-(4) и независимости последовательностей $\left\{x_{t}\right\},\left\{\xi_{t}\right\},\left\{\gamma_{t}\right\}$ имеем цепочку равенств:

$$
\begin{aligned}
p_{v_{0}, v_{1}}^{(2)}=\mathbf{P}\left\{Y_{t}=1, Y_{t+1}=1\right\}= & \\
= & \mathbf{P}\left\{\gamma_{t}=0, \gamma_{t+1}=0\right\} \mathbf{P}\left\{x_{t}=v_{0}, x_{t+1}=v_{1} \mid \gamma_{t}=0, \gamma_{t+1}=0\right\}+ \\
& +\mathbf{P}\left\{\gamma_{t}=0, \gamma_{t+1}=1\right\} \mathbf{P}\left\{x_{t}=v_{0}, \xi_{\tau_{t+1}}=v_{1} \mid \gamma_{t}=0, \gamma_{t+1}=1\right\}+ \\
& +\mathbf{P}\left\{\gamma_{t}=1, \gamma_{t+1}=0\right\} \mathbf{P}\left\{\xi_{\tau_{t}}=v_{0}, x_{t+1}=v_{1} \mid \gamma_{t}=1, \gamma_{t+1}=0\right\}+ \\
+ & \mathbf{P}\left\{\gamma_{t}=1, \gamma_{t+1}=1\right\} \mathbf{P}\left\{\xi_{\tau_{t}}=v_{0}, \xi_{\tau_{t+1}}=v_{1} \mid \gamma_{t}=1, \gamma_{t+1}=1\right\}= \\
& =(1-\delta)^{2} p_{v_{0}, v_{1}} / 2+\delta(1-\delta)\left(\theta_{v_{1}}+\theta_{v_{0}}\right) / 2+\delta^{2} \theta_{v_{0}} \theta_{v_{1}},
\end{aligned}
$$

что совпадает с (9). Просуммировав по компоненте $v_{1} \in V$, получаем: $p_{v_{0}}^{(1)}=$ $\sum_{v_{1}=0}^{1} p_{v_{0}, v_{1}}^{(2)}=(1-\delta)^{2} / 2+\delta(1-\delta)\left(1 / 2+\theta_{v_{0}}\right)+\delta^{2} \theta_{v_{0}}=(1-\delta) / 2+\delta \theta_{v_{0}}$, что совпадает с (8).

Соотношение (10) выводится аналогично.

Заметим, что если $\theta_{0}=\theta_{1}=1 / 2$, то $p_{v}^{(1)}$ не зависит от $v \in V: p_{0}^{(1)}=p_{1}^{(1)}=1 / 2$. Это означает, что одномерное распределение вероятностей стегопоследовательности $\left\{Y_{t}\right\}$ не несет никакой информации о параметрах модели $\varepsilon, \delta$. Отметим еще, что стегопоследовательность $\left\{Y_{t}\right\}$ при $\delta>0$, вообще говоря, не является цепью Маркова 1-го порядка, так как в общем случае в силу (8)-(10) нарушается марковское свойство: $p_{v_{0}, v_{1}, v_{2}}^{(3)} \neq p_{v_{0}, v_{1}}^{(2)} \cdot p_{v_{1}, v_{2}}^{(2)} / p_{v_{1}}^{(1)}$.

Будем далее рассматривать наиболее трудный для стегоаналитика случай, когда одномерные распределения вероятностей контейнера и сообщения совпадают: $\theta_{0}=\theta_{1}=1 / 2$. Тогда согласно $(9)$ двумерное $(s=2)$ распределение вероятностей стегопоследовательности $\left\{Y_{t}\right\}$ имеет вид $p_{v_{0}, v_{1}}^{(2)}=(1-\delta)^{2}\left(1+(-1)^{v_{0}+v_{1}} \varepsilon\right) / 4+\delta(2-\delta) / 4$, и для определения $\varepsilon, \delta$ по $\left\{p_{v_{0}, v_{1}}^{(2)}\right\}$ имеем систему уравнений:

$$
\left\{\begin{array}{l}
p_{0,0}^{(2)}=p_{1,1}^{(2)}=1 / 4+(1-\delta)^{2} \varepsilon / 4, \\
p_{0,1}^{(2)}=p_{1,0}^{(2)}=1 / 4-(1-\delta)^{2} \varepsilon / 4 .
\end{array}\right.
$$


Обозначим через $f_{v_{0} \ldots v_{s-1}}=\sum_{t=1}^{T-s+1} I\left\{Y_{t}^{t+s-1}=v_{0}^{s-1}\right\}$ абсолютную частоту $s$ граммы, $\left(v_{0}, \ldots, v_{s-1}\right) \in V_{s}$. Из $(11)$ при известном $\delta<1$ получаем подстановочную (plug-in) оценку параметра $\varepsilon$, являющуюся функцией частот биграмм $\left\{f_{v_{0} v_{1}}\right\}$ при $s=2$ :

$$
\hat{\varepsilon}=g_{0}\left(\frac{f_{00}}{T-1}, \frac{f_{11}}{T-1}, \frac{f_{01}}{T-1}, \frac{f_{10}}{T-1}\right)=\frac{f_{00}+f_{11}-f_{01}-f_{10}}{(1-\delta)^{2}(T-1)} .
$$

Теорема 2. Для модели вкраплений (2)-(4) при $\delta<1$ статистика (12) является несмещенной и состоятельной при $T \rightarrow \infty$ оценкой параметра модели $\varepsilon: \mathbf{E}\{\hat{\varepsilon}\}=\varepsilon$, $\hat{\varepsilon} \stackrel{P}{\rightarrow} \varepsilon$.

Доказательство. В силу (9) для модели (2)-(4) имеем:

$$
\mathbf{E}\left\{f_{v_{0} v_{1}}\right\}=(T-1) p_{v_{0} v_{1}}^{(2)}=(T-1)\left(1+(-1)^{v_{0}+v_{1}}(1-\delta)^{2} \varepsilon\right) / 4 .
$$

Из (13) следует, что оценка $\hat{\varepsilon}$ является несмещенной:

$$
\mathbf{E}\{\hat{\varepsilon}\}=\mathbf{E}\left\{\frac{f_{00}+f_{11}-f_{01}-f_{10}}{(1-\delta)^{2}(T-1)}\right\}=\frac{(T-1)\left(2+2(1-\delta)^{2} \varepsilon-\left(2-2(1-\delta)^{2} \varepsilon\right)\right)}{4(1-\delta)^{2}(T-1)}=\varepsilon .
$$

В силу принятых обозначений, группируя слагаемые, получаем:

$$
\begin{gathered}
\mathbf{E}\left\{f_{11}^{2}\right\}=\sum_{t, t^{\prime}=1}^{T-1} \mathbf{P}\left\{Y_{t}=Y_{t+1}=Y_{t^{\prime}}=Y_{t^{\prime}+1}=1\right\}=(T-1) \mathbf{P}\left\{Y_{t}=Y_{t+1}=1\right\}+ \\
+2(T-2) \mathbf{P}\left\{Y_{t}=Y_{t+1}=Y_{t+2}=1\right\}+2 \sum_{\tau=2}^{T-2}(T-1-\tau) \mathbf{P}\left\{Y_{t}=Y_{t+1}=Y_{t+\tau}=Y_{t+\tau+1}=1\right\} .
\end{gathered}
$$

С учетом модели (2)-(4) при $\tau \geqslant 2$ находим:

$$
\begin{gathered}
\mathbf{P}\left\{Y_{t}=Y_{t+1}=Y_{t+\tau}=Y_{t+\tau+1}=1\right\}= \\
=\sum_{u_{0}, \ldots, u_{3} \in V_{4}} \mathbf{P}\left\{\gamma_{t}=u_{0}, \gamma_{t+1}=u_{1}, \gamma_{t+\tau}=u_{2}, \gamma_{t+\tau+1}=u_{3}\right\} \times \\
\times \mathbf{P}\left\{Y_{t}=Y_{t+1}=Y_{t+\tau}=Y_{t+\tau+1}=1 \mid \gamma_{t}=u_{0}, \gamma_{t+1}=u_{1}, \gamma_{t+\tau}=u_{2}, \gamma_{t+\tau+1}=u_{3}\right\}= \\
=(1-\delta)^{4}\left(1+2 \varepsilon+\varepsilon^{2}+\varepsilon^{\tau-1}+2 \varepsilon^{\tau}+\varepsilon^{\tau+1}\right) / 16+2 \delta(1-\delta)^{3}\left(2+2 \varepsilon+\varepsilon^{2}+\varepsilon^{\tau-1}+2 \varepsilon^{\tau}+\varepsilon^{\tau+1}\right) / 16+ \\
\delta^{2}(1-\delta)^{2}\left(6+2 \varepsilon+\varepsilon^{\tau-1}+2 \varepsilon^{\tau}+\varepsilon^{\tau+1}\right) / 16+4 \delta^{3}(1-\delta) / 16+\delta^{4} / 16= \\
=\left(1+2 \varepsilon(1-\delta)^{2}+\varepsilon^{2}(1-\delta)^{4}+(1+\varepsilon)^{2}(1-\delta)^{2} \varepsilon^{\tau-1}\right) / 16= \\
=\left(1+\varepsilon(1-\delta)^{2}\right)^{2} / 16+(1+\varepsilon)^{2}(1-\delta)^{2} \varepsilon^{\tau-1} / 16 .
\end{gathered}
$$

Учитывая (13), проверим выполнение достаточного условия Маркова закона больших чисел [14]:

$$
\begin{gathered}
\mathbf{D}\left\{f_{11}\right\} /(T-1)^{2}=\mathbf{E}\left\{f_{11}^{2}\right\} /(T-1)^{2}-\mathbf{E}^{2}\left\{f_{11}\right\} /(T-1)^{2}=\mathbf{E}\left\{f_{11}^{2}\right\} /(T-1)^{2}-\left(p_{11}^{(2)}\right)^{2}= \\
=\mathbf{P}\left\{Y_{t}=Y_{t+1}=1\right\} /(T-1)+2(T-1)^{-2}\left((T-2) \mathbf{P}\left\{Y_{t}=Y_{t+1}=Y_{t+2}=1\right\}+\right. \\
\left.+\left(p_{11}^{(2)}\right)^{2} \sum_{\tau=2}^{T-2}(T-1-\tau)+(1+\varepsilon)^{2}(1-\delta)^{2} \sum_{\tau=2}^{T-2}(T-1-\tau) \varepsilon^{\tau-1} / 16\right)-\left(p_{11}^{(2)}\right)^{2} \underset{T \rightarrow \infty}{\longrightarrow} 0 .
\end{gathered}
$$


Следовательно, $f_{11} /(T-1) \stackrel{\mathbf{P}}{\rightarrow} p_{11}^{(2)}$. Аналогично можно показать, что $f_{v_{0} v_{1}} /(T-1) \stackrel{\mathbf{P}}{\rightarrow}$ $p_{v_{0} v_{1}}^{(2)}$ при всех $v_{0}, v_{1} \in V$. Тогда из свойства непрерывности функции $g_{0}\left(z_{1}, z_{2}, z_{3}, z_{4}\right)$, входящей в (12), и свойств функциональных преобразований случайных последовательностей [14] следует, что оценка $\hat{\varepsilon}$ состоятельна.

Из (10) при $s=3$ имеем: $p_{v_{0}, v_{1}, v_{2}}^{(3)}=1 / 8+(1-\delta)^{2}\left((-1)^{v_{1}}\left((-1)^{v_{0}}+(-1)^{v_{2}}\right)+\right.$ $\left.(-1)^{v_{0}+v_{2}} \varepsilon\right) \varepsilon / 8$. Тогда для определения $\varepsilon, \delta$ по $\left\{p_{v_{0}, v_{1}, v_{2}}^{(3)}\right\}$ можно составить систему уравнений

$$
\left\{\begin{array}{l}
p_{0,0,0}^{(3)}=p_{1,1,1}^{(3)}=1 / 8+(1-\delta)^{2}(2+\varepsilon) \varepsilon / 8 \\
p_{0,1,0}^{(3)}=p_{1,0,1}^{(3)}=1 / 8+(1-\delta)^{2}(-2+\varepsilon) \varepsilon / 8 \\
p_{0,0,1}^{(3)}=p_{1,1,0}^{(3)}=p_{0,1,1}^{(3)}=p_{1,0,0}^{(3)}=1 / 8-(1-\delta)^{2} \varepsilon^{2} / 8
\end{array}\right.
$$

Из системы уравнений (14) следует соотношение

$$
(1-\delta)^{2}=\left(f_{000}+f_{111}+f_{010}+f_{101}-f_{001}-f_{110}-f_{011}-f_{100}\right) / \varepsilon^{2}(T-2) .
$$

Подставляя сюда оценку $\hat{\varepsilon}$, определяемую (12), и учитывая ограничение: $\delta \in[0,1]$, получаем статистическую оценку для $\delta$ :

$$
\begin{gathered}
\hat{\delta}=1-\sqrt{\max \left\{0, \min \left\{1, g_{1}\right\}\right\}}, \\
g_{1}=\frac{\left(f_{00}+f_{11}-f_{01}-f_{10}\right)^{2}(T-2)}{\left(f_{000}+f_{111}+f_{010}+f_{101}-f_{001}-f_{110}-f_{011}-f_{100}\right)(T-1)^{2}} .
\end{gathered}
$$

Теорема 3. Для модели вкраплений (2)-(4) статистика (16) является состоятельной при $T \rightarrow \infty$ оценкой параметра модели $\delta: \hat{\delta} \stackrel{\mathbf{P}}{\rightarrow} \delta$.

Доказательство. Проводится аналогично доказательству теоремы 2.

Исследуем теперь корреляционные свойства стегопоследовательности $\left\{Y_{t}\right\}$.

Теорема 4. Если имеет место модель побитового вкрапления (2)-(4) и $\theta_{0}=\theta_{1}=$ $1 / 2$, то коррелячионная функиия $\rho_{l}$ стегопоследовательности $\left\{Y_{t}\right\}$ имеет вид

$$
\rho_{l}=\operatorname{Corr}\left\{Y_{t}, Y_{t+l}\right\}=(1-\delta)^{2} \varepsilon^{|l|}, l= \pm 1, \pm 2, \ldots
$$

Доказательство. Для модели (2)-(4) в силу (8) имеем: $\mathbf{E}\left\{Y_{t}\right\}=\mathbf{P}\left\{Y_{t}=1\right\}=$ $1 / 2, \mathbf{D}\left\{Y_{t}\right\}=1 / 4$. С учетом леммы 1 и доказательства теоремы 1 справедливо соотношение

$$
\begin{aligned}
\mathbf{E}\left\{Y_{t} Y_{t+l}\right\}=\mathbf{P}\left\{Y_{t}\right. & \left.=1, Y_{t+l}=1\right\}= \\
& =(1-\delta)^{2}\left(1+\varepsilon^{|l|}\right) / 4+\delta(1-\delta) \theta_{1}+\delta^{2} \theta_{1}^{2}=(1-\delta)^{2} \varepsilon^{|l|} / 4+1 / 4 .
\end{aligned}
$$

Подставляя эти выражения в $\rho_{l}=\left(\mathbf{E}\left\{Y_{t} Y_{t+l}\right\}-\mathbf{E}^{2}\left\{Y_{t}\right\}\right) / \mathbf{D}\left\{Y_{t}\right\}$, получаем (17).

Следствие 1. В условиях теоремы 4 оценки параметров $\varepsilon, \delta$, основанные на корреляииях (17), имеют вид

$$
\hat{\varepsilon}=\min \left\{1, \max \left\{-1, \hat{\rho}_{2} / \hat{\rho}_{1}\right\}\right\}, \hat{\delta}=1-\sqrt{\max \left\{0, \min \left\{1, \hat{\rho}_{1}^{2} / \hat{\rho}_{2}\right\}\right.},
$$

где $\hat{\rho}_{l}=\frac{4}{T-l} \sum_{t=1}^{T-l} I\left\{Y_{t}=1\right\} I\left\{Y_{t+l}=1\right\}-1$ - выборочная корреляиионная функиия. Следствие 2. В условиях теоремы 4 статистики (18) являются состоятельными при $T \rightarrow \infty$ оценками параметров модели $\varepsilon, \delta$.

Доказательство. Аналогично доказательству теоремы 3. 


\section{4. Оценки максимального правдоподобия парамет- ров $\varepsilon, \delta$ для модели побитового вкрапления}

По наблюдаемой стегопоследовательности $y_{1}^{T}=\left(y_{1}, \ldots, y_{T}\right) \in V_{T}$ построим функцию правдоподобия.

Разобьем множество $V_{t}$ двоичных $t$-мерных векторов на $t+1$ непересекающихся подмножеств:

$$
V_{t}=\Gamma_{0}^{(t)} \cup \Gamma_{1}^{(t)} \cup \ldots \cup \Gamma_{t}^{(t)},
$$

где

$$
\begin{aligned}
& \Gamma_{0}^{(t)}=\left\{u_{1}^{t} \in V_{t}: u_{t}=1\right\}, \\
& \Gamma_{1}^{(t)}=\left\{u_{1}^{t} \in V_{t}: u_{t}=u_{t-1}=0\right\}, \\
& \Gamma_{j}^{(t)}=\left\{u_{1}^{t} \in V_{t}: u_{t}=0, u_{t-1}=\ldots=u_{t-j+1}=1, u_{t-j}=0\right\}, 1<j<t, \\
& \Gamma_{t}^{(t)}=\left\{u_{1}^{t} \in V_{t}: u_{t}=0, u_{t-1}=\ldots=u_{1}=1\right\} .
\end{aligned}
$$

Разбиение (19), (20) порождает разбиение всевозможных траекторий фрагментов ключевой последовательности $\gamma_{1}^{t}=u_{1}^{t} \in V_{t}$ (см. рис. 1).

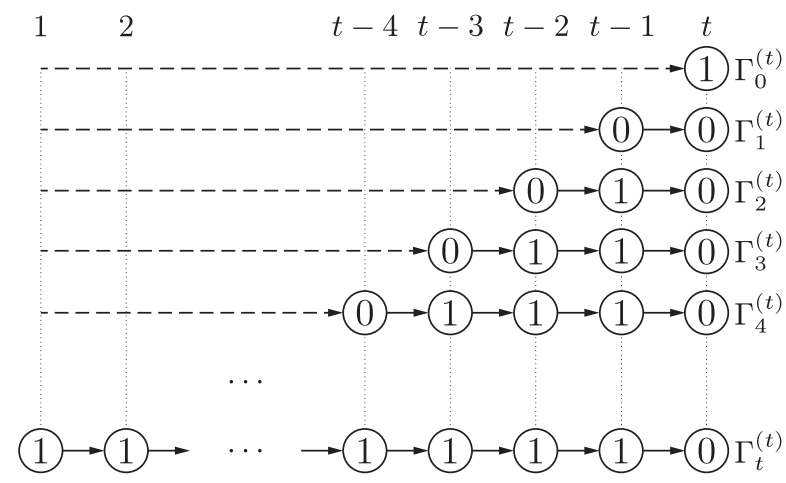

Рис. 1. Разбиение траекторий $\gamma_{1}^{t}$; штриховая стрелка - произвольная траектория.

Используя разбиение (19),(20), определим функции двоичных переменных $u_{1}^{t} \in V_{t}, y_{1}^{t} \in V_{t}(t \geqslant 1):$

$$
\varphi_{t}\left(u_{1}^{t}, y_{1}^{t}\right)=\left\{\begin{array}{l}
\theta_{y_{t}}, u_{1}^{t} \in \Gamma_{0}^{(t)}, \\
\frac{1}{2}\left(1+(-1)^{y_{t-j}+y_{t}} \varepsilon^{j}\right), u_{1}^{t} \in \Gamma_{j}^{(t)}, 1 \leqslant j<t, \\
\frac{1}{2}, u_{1}^{t} \in \Gamma_{t}^{(t)} .
\end{array}\right.
$$

Теорема 5. Если имеет место модель побитового вкрапления (2)-(4), то функиця правдоподобия $L\left(\varepsilon, \delta ; y_{1}^{T}\right)$ для наблюдаемой стегопоследовательности $y_{1}^{T} \in V_{T}$ имеет вид:

$$
L\left(\varepsilon, \delta ; y_{1}^{T}\right)=\mathbf{P}\left\{Y_{1}^{T}=y_{1}^{T}\right\}=\sum_{u_{1}^{T} \in V_{T}} \delta^{w\left(u_{1}^{T}\right)}(1-\delta)^{T-w\left(u_{1}^{T}\right)} \prod_{t=1}^{T} \varphi_{t}\left(u_{1}^{t}, y_{1}^{t}\right) .
$$


Доказательство. С учетом (3), (4) и принятых обозначений имеем:

$$
\begin{aligned}
L\left(\varepsilon, \delta ; y_{1}^{T}\right)=\mathbf{P}\left\{Y_{1}^{T}=y_{1}^{T}\right\}=\sum_{u_{1}^{T} \in V_{T}} \mathbf{P}\left\{\gamma_{1}^{T}=u_{1}^{T}\right\} L_{u_{1}, \ldots, u_{T}}\left(\varepsilon ; y_{1}^{T}\right)= \\
=\sum_{u_{1}^{T} \in V_{T}} \delta^{w\left(u_{1}^{T}\right)}(1-\delta)^{T-w\left(u_{1}^{T}\right)} L_{u_{1}, \ldots, u_{T}}\left(\varepsilon ; y_{1}^{T}\right)=\mathbf{E}\left\{L_{\gamma_{1}, \ldots, \gamma_{T}}\left(\varepsilon ; y_{1}^{T}\right)\right\},
\end{aligned}
$$

где $L_{u_{1}, \ldots, u_{T}}\left(\varepsilon ; y_{1}^{T}\right)=\mathbf{P}\left\{Y_{1}^{T}=y_{1}^{T} \mid \gamma_{1}^{T}=u_{1}^{T}\right\}$ - условная функция правдоподобия (при условии, что стегоключ $\left.\gamma_{1}^{T}=u_{1}^{T} \in V_{T}\right)$ f:

$$
\begin{gathered}
L_{u_{1}, \ldots, u_{T}}\left(\varepsilon ; y_{1}^{T}\right)=\mathbf{P}\left\{Y_{1}=y_{1} \mid \gamma_{1}=u_{1}\right\} \mathbf{P}\left\{Y_{2}=y_{2} \mid Y_{1}=y_{1}, \gamma_{1}^{2}=u_{1}^{2}\right\} \times \cdots \\
\cdots \times \mathbf{P}\left\{Y_{T}=y_{T} \mid Y_{1}^{T-1}=y_{1}^{T-1}, \gamma_{1}^{T}=u_{1}^{T}\right\}= \\
=\mathbf{P}\left\{Y_{1}=y_{1} \mid \gamma_{1}=u_{1}\right\} \prod_{t=2}^{T} \mathbf{P}\left\{Y_{t}=y_{t} \mid Y_{1}^{t-1}=y_{1}^{t-1}, \gamma_{1}^{t}=u_{1}^{t}\right\} .
\end{gathered}
$$

Первый сомножитель в правой части этого равенства в силу (2)-(4) и независимости $\xi_{1}, \gamma_{1}$ равен

$$
\mathbf{P}\left\{Y_{1}=y_{1} \mid \gamma_{1}=u_{1}\right\}=\left\{\begin{array}{l}
\mathbf{P}\left\{\xi_{1}=y_{1} \mid \gamma_{1}=1\right\}=\theta_{y_{1}}, u_{1}=1, \\
\mathbf{P}\left\{x_{1}=y_{1} \mid \gamma_{1}=0\right\}=1 / 2, u_{1}=0 .
\end{array}\right.
$$

Во втором сомножителе, используя лемму 1, обозначения (19)-(21), независимость $\left\{x_{t}\right\},\left\{\gamma_{t}\right\}$ и $\left\{\xi_{t}\right\}$, получаем для $t \geqslant 2$ :

$$
\begin{aligned}
& \mathbf{P}\left\{Y_{t}=\right.\left.y_{t} \mid Y_{1}^{t-1}=y_{1}^{t-1}, \gamma_{1}^{t}=u_{1}^{t}\right\}=\varphi_{t}\left(u_{1}^{t}, y_{1}^{t}\right)= \\
&=\left\{\begin{array}{l}
\mathbf{P}\left\{\xi_{t}=y_{t} \mid Y_{1}^{t-1}=y_{1}^{t-1}, \gamma_{t}=1, \gamma_{1}^{t-1}=u_{1}^{t-1}\right\}, u_{1}^{t} \in \Gamma_{0}^{(t)}, \\
\mathbf{P}\left\{x_{t}=y_{t} \mid \xi_{t-j+1}^{t-1}=y_{t-j+1}^{t-1}, x_{t-j}=y_{t-j}, Y_{1}^{t-j-1}=y_{1}^{t-j-1}, \gamma_{1}^{t}=u_{1}^{t}\right\}, \\
u_{1}^{t} \in \Gamma_{j}^{(t)}, 1 \leqslant j<t,
\end{array}\right. \\
& \mathbf{P}\left\{x_{t}=y_{t} \mid \xi_{1}^{t-1}=y_{1}^{t-1}, \gamma_{t}=0, \gamma_{t-1}=\ldots=\gamma_{1}=1\right\}, u_{1}^{t} \in \Gamma_{t}^{(t)} .
\end{aligned}
$$

Объединяя эти выражения, приходим к (22).

Следствие 3. Оценки максимального правдоподобия (МП-оценки) $\hat{\varepsilon}, \hat{\delta}$ параметров $\varepsilon, \delta$ определяются как решение экстремальной задачи

$$
L\left(\varepsilon, \delta ; y_{1}^{T}\right)=\mathbf{E}\left\{L_{\gamma_{1}, \ldots, \gamma_{T}}\left(\varepsilon ; y_{1}^{T}\right)\right\} \rightarrow \max _{\varepsilon \in(-1,1), \delta \in[0,1]} .
$$

Следствие 4. $B$ частном случае отсутствия вкраплений $\left(\delta=0, \gamma_{t} \equiv 0\right)$ МПоценка параметра є совпадает с ранее полученной частотной оценкой (12):

$$
\hat{\varepsilon}_{0}=\frac{f_{00}+f_{11}-f_{01}-f_{10}}{T-1}
$$

эта оценка является несмещенной и при $T \rightarrow \infty, \varepsilon \in(-1,1)$, имеет асимптотически нормальное распределение вероятностей: $\mathfrak{L}\left\{\sqrt{T-1}\left(\hat{\varepsilon}_{0}-\varepsilon\right)\right\} \rightarrow N\left(0,1-\varepsilon^{2}\right)$. 
Доказательство. МП-оценка находится максимизацией логарифмической функции правдоподобия:

$$
\begin{aligned}
& l\left(\varepsilon, 0 ; y_{1}^{T}\right) \stackrel{\text { def }}{=} \log L\left(\varepsilon, 0 ; y_{1}^{T}\right)=\log L_{0, \ldots, 0}\left(\varepsilon ; y_{1}^{T}\right)=\log \mathbf{P}\left\{Y_{1}^{T}=y_{1}^{T} \mid \gamma_{1}=0, \ldots, \gamma_{T}=0\right\}= \\
& =\log \mathbf{P}\left\{x_{1}^{T}=y_{1}^{T}\right\}=\log \pi_{y_{1}}+\sum_{t=2}^{T} \log p_{y_{t-1}, y_{t}}=\log \pi_{y_{1}}+\sum_{v_{0}, v_{1}=0}^{1} f_{v_{0} v_{1}} \log p_{v_{0}, v_{1}}(\varepsilon)= \\
& =-\log 2+\left(f_{00}+f_{11}\right) \log (1+\varepsilon)+\left(f_{01}+f_{10}\right) \log (1-\varepsilon)-(T-1) \log 2 \rightarrow \max _{\varepsilon \in(-1,1)} .
\end{aligned}
$$

Из уравнения $l^{\prime}\left(\varepsilon, 0 ; y_{1}^{T}\right)=0$ получаем единственное решение $(24)$, которое соответствует точке максимума.

Несмещенность оценки следует из теоремы 2 при $\delta=0$.

Используя теоремы о функциональных преобразованиях асимптотически нормальных статистик из [14], получаем, что оценка $\hat{\varepsilon}$ асимптотически нормальна при $T \rightarrow \infty$ и что $1-\varepsilon^{2}$ является асимптотической дисперсией этой оценки.

Из-за существенной нелинейности решение экстремальной задачи (23) возможно только численными методами (например, методом табулирования $L\left(\varepsilon, \delta ; y_{1}^{T}\right)$ на сетке или методом градиентного спуска), требующими вычисления функции правдоподобия для заданной последовательности точек. Согласно определению (22), вычисление одного значения функции правдоподобия $L\left(\varepsilon, \delta ; y_{1}^{T}\right)$ при фиксированных параметрах $\varepsilon, \delta$ имеет вычислительную сложность порядка $O\left(T 2^{T}\right)$, т. е. экспоненциальную сложность относительно длины стегопоследовательности.

Отметим, что для численной оценки функции правдоподобия можно применить метод Монте-Карло: $\tilde{L}\left(\varepsilon, \delta, K ; y_{1}^{T}\right)=\frac{1}{K} \sum_{k=1}^{K} L_{u_{1}^{(k)}, \ldots, u_{T}^{(k)}}\left(\varepsilon ; y_{1}^{T}\right)$, где $K$ - число имитаций, $\left(u_{1}^{(k)}, \ldots, u_{T}^{(k)}\right) \in V_{T}-k$-я реализация стегоключа $\gamma_{1}^{T}, \mathbf{P}\left\{\gamma=\left(u_{1}^{(k)}, \ldots, u_{T}^{(k)}\right)\right\}=$ $\delta^{\sum_{t=1}^{T} u_{t}^{(k)}}(1-\delta)^{T-\sum_{t=1}^{T} u_{t}^{(k)}}$. Вычислительная сложность этого алгоритма имеет порядок $O(K T)$. При этом имеет место случайная ошибка вычисления значения функции правдоподобия, для которой среднеквадратическое отклонение имеет порядок $O(1 / \sqrt{K})$.

\section{5. Оценивание параметров $\varepsilon, \delta$ для модели блочного вкрапления}

Использование модели блочного вкрапления (2)-(3), (5) позволяет построить алгоритмы вычисления функции правдоподобия и оценок максимального правдоподобия $\hat{\varepsilon}, \hat{\delta}$, имеющие полиномиальную относительно длины стегопоследовательности $T$ сложность.

Лемма 2. Если имеет место блочная модель (5) ключевой последовательности $\left\{\gamma_{t}\right\}$, mo nрu $t>3$

$$
\mathbf{P}\left\{\gamma_{1}^{t} \in \bigcup_{j>3} \Gamma_{j}^{(t)}\right\}=0,
$$

u стегопоследовательность $\left\{Y_{t}\right\}$, определяемая (2)-(3), при фиксированной последовательности $\left\{\gamma_{t}\right\}$ является управляемой цепъю Маркова условного порядка 
$s_{t} \in\{0,1,2,3\}$, причем порядок $s_{t}$ зависит от ключевой последовательности $\left\{\gamma_{t}\right\}:$

$$
s_{t}=j, \text { если } u_{1}^{t} \in \Gamma_{j}^{(t)} .
$$

Доказательство. Соотношение (25) вытекает из (5) и обозначений (20). Для доказательства (26) достаточно проверить марковское свойство последовательности $Y_{t}$ при фиксированной управляющей последовательности $\gamma_{t}$ аналогично ходу доказательства теоремы 5 :

$$
\begin{aligned}
\mathbf{P}\left\{Y_{t}=y_{t} \mid Y_{1}^{t-1}=y_{1}^{t-1}, \gamma_{1}^{t}=\right. & \left.u_{1}^{t}\right\}=p_{y_{t-3}, y_{t-2}, y_{t-1}, y_{t}}\left(u_{t-3}^{t}\right)= \\
& =\left\{\begin{array}{l}
\theta_{y_{t}}, u_{1}^{t} \in \Gamma_{0}^{(t)}, \\
\frac{1}{2}\left(1+(-1)^{y_{t-j}+y_{t}} \varepsilon^{j}\right), u_{1}^{t} \in \Gamma_{j}^{(t)}, 1 \leqslant j \leqslant 3 .
\end{array}\right.
\end{aligned}
$$

Следствие 5. Если $\theta_{0}=\theta_{1}=1 / 2$, то $8 \times 2$ - матрица усредненных по $\gamma_{t-3}^{t}$ вероятностей одношаговых переходов управляемой иепи Маркова 3-го порядка имеет вид

$$
\bar{P}_{t}=\left(\bar{p}_{y_{t-3}, y_{t-2}, y_{t-1}, y_{t}}\right)=\left\{\begin{array}{l}
\left(\begin{array}{l}
C A_{2 k}, \\
C A_{2 k-1},
\end{array} 1_{8}-C A_{2 k}\right), t=2 k, k \in \mathbb{N}, \\
\left.C A_{2 k-1}\right), t=2 k-1, k \in \mathbb{N},
\end{array}\right.
$$

где $1_{8}=\left(\begin{array}{llllllll}1 & 1 & 1 & 1 & 1 & 1 & 1 & 1\end{array}\right)^{\prime}$,

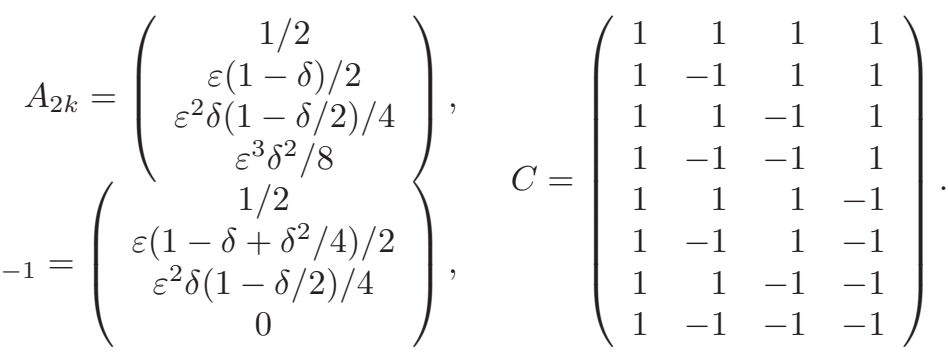

Доказательство. Имеем по определению:

$$
\bar{p}_{y_{t-3}, y_{t-2}, y_{t-1}, y_{t}}=\sum_{j=0}^{3} \mathbf{P}\left\{\gamma_{1}^{t} \in \Gamma_{j}^{(t)}\right\} p_{y_{t-3}, y_{t-2}, y_{t-1}, y_{t}}\left(u_{t-3}^{t}\right) .
$$

Подставляя сюда (27) и следующие из (5) значения вероятностей

$$
\mathbf{P}\left\{\gamma_{1}^{2 k} \in \Gamma_{j}^{(2 k)}\right\}=\left\{\begin{array}{l}
\frac{\delta}{2}, j=0, \\
1-\delta, j=1, \\
\delta \frac{1-\frac{\delta}{2}}{2}, j=2, \\
\frac{\delta^{2}}{4}, j=3,
\end{array} \quad \mathbf{P}\left\{\gamma_{1}^{2 k-1} \in \Gamma_{j}^{(2 k-1)}\right\}=\left\{\begin{array}{l}
\frac{\delta}{2}, j=0, \\
1-\delta+\frac{\delta^{2}}{4}, j=1, \\
\delta \frac{1-\frac{\delta}{2}}{2}, j=2, \\
0, j=3,
\end{array}\right.\right.
$$

получаем (28).

Заметим, что аналогично (12),(16) соотношение (28) удобно использовать при построении частотных оценок параметров $\varepsilon, \delta$ для блочной модели (5) ключевой последовательности $\left\{\gamma_{t}\right\}$. 
Используя лемму 2, построим полиномиальный относительно $T$ алгоритм вычисления функции правдоподобия для блочной модели стегоключа (5), основанный на алгоритме "Forward" [16].

Для этого вначале аналогично (22) построим функцию правдоподобия для блочной модели стегоключа (5):

$$
L\left(\varepsilon, \delta ; y_{1}^{T}\right)=\sum_{u_{1}^{T} \in V_{T}} I\left\{b_{2}\left(u_{1}^{T}\right)=0\right\}(1-\delta)^{b_{0}\left(u_{1}^{T}\right)}(\delta / 2)^{b_{1}\left(u_{1}^{T}\right)} \prod_{t=1}^{T} \varphi_{t}\left(u_{1}^{t}, y_{1}^{t}\right),
$$

где $b_{k}\left(u_{1}^{T}\right)=\sum_{t=1}^{T / 2} I\left\{w\left(u_{2 t-1}, u_{2 t}\right)=k\right\}, k \in\{0,1,2\}$.

Заметим, что согласно лемме 2 в сумме (29) исключаются слагаемые, для которых $u_{1}^{t} \in \bigcup_{j>3} \Gamma_{j}^{(t)}$, поэтому функция $\varphi_{t}\left(u_{1}^{t}, y_{1}^{t}\right)=p_{y_{t-3}, y_{t-2}, y_{t-1}, y_{t}}\left(u_{t-3}^{t}\right)$ фактически зависит только от $u_{t-3}^{t}, y_{t-3}^{t}$.

Пусть $r \in \mathbb{N}$ - вспомогательный параметр, $\alpha_{t}\left(u_{0}, \ldots, u_{r-1}\right)=\mathbf{P}\left\{Y_{1}=y_{1}, \ldots, Y_{t}=\right.$ $\left.y_{t}, \gamma_{t-r+1}=u_{0}, \ldots, \gamma_{t}=u_{r-1}\right\}, t>r,-$ это вероятность наблюдения последовательности $y_{1}, \ldots, y_{t}$ до момента времени $t$ и состояний стегоключа $u_{0}, \ldots, u_{r-1}$ в моменты времени $t-r+1, \ldots, t$.

Теорема 6. Если имеет место модель блочного вкрапления (2)-(3),(5), $r \geqslant 3$, то для $\alpha_{t}\left(u_{0}, \ldots, u_{r-1}\right)$, при $t=r+1, r+2, \ldots, T$ справедливо рекуррентное соотношение

$$
\alpha_{t}\left(u_{0}, \ldots, u_{r-1}\right)=q_{t, u_{r-2}, u_{r-1}} \sum_{u_{-1} \in V} \alpha_{t-1}\left(u_{-1}, \ldots, u_{r-2}\right) p_{y_{t-3}, y_{t-2}, y_{t-1}, y_{t}}\left(u_{r-4}^{r-1}\right),
$$

где $\left\{q_{t, i, j}\right\}$ определяются формулой $(6)$.

Доказательство. По построению $\gamma_{t}, \gamma_{t^{\prime}}$ зависимы лишь тогда, когда они принадлежат одному и тому же блоку, т.е. $t=2 k-1, t^{\prime}=2 k$ или $t=2 k, t^{\prime}=2 k-1$ при нектором $k \in \mathbb{N}$, поэтому с учетом (5) по формуле полной вероятности имеем:

$$
\begin{aligned}
& \alpha_{t}\left(u_{0}, \ldots, u_{r-1}\right)=\mathbf{P}\left\{Y_{1}^{t}=y_{1}^{t}, \gamma_{t-r+1}=u_{0}, \ldots, \gamma_{t}=u_{r-1}\right\}= \\
& =\sum_{u_{-1} \in V} \mathbf{P}\left\{Y_{1}^{t}=y_{1}^{t}, \gamma_{t-r}=u_{-1}, \gamma_{t-r+1}=u_{0}, \ldots, \gamma_{t}=u_{r-1}\right\}= \\
& =\sum_{u_{-1} \in V} \mathbf{P}\left\{Y_{1}^{t-1}=y_{1}^{t-1}, \gamma_{t-r}=u_{-1}, \gamma_{t-r+1}=u_{0}, \ldots, \gamma_{t-1}=u_{r-2}\right\} \times \\
& \times \mathbf{P}\left\{\gamma_{t}=u_{r-1} \mid Y_{1}^{t-1}=y_{1}^{t-1}, \gamma_{t-r}=u_{-1}, \gamma_{t-r+1}=u_{0}, \ldots, \gamma_{t-1}=u_{r-2}\right\} \times \\
& \times \mathbf{P}\left\{Y_{t}=y_{t} \mid Y_{1}^{t-1}=y_{1}^{t-1}, \gamma_{t-r}=u_{-1}, \gamma_{t-r+1}=u_{0}, \ldots, \gamma_{t}=u_{r-1}\right\}= \\
& \quad=\sum_{u_{-1} \in V} \alpha_{t-1}\left(u_{-1}, u_{0}, \ldots, u_{r-2}\right) \mathbf{P}\left\{\gamma_{t}=u_{r-1} \mid \gamma_{t-1}=u_{r-2}\right\} \times \\
& \quad \times \mathbf{P}\left\{Y_{t}=y_{t} \mid Y_{1}^{t-1}=y_{1}^{t-1}, \gamma_{t-r}=u_{-1}, \gamma_{t-r+1}=u_{0}, \ldots, \gamma_{t}=u_{r-1}\right\} .
\end{aligned}
$$

В силу 2 справедливо соотношение

$$
\mathbf{P}\left\{Y_{t}=y_{t} \mid Y_{1}^{t-1}=y_{1}^{t-1}, \gamma_{t-r}=u_{-1}, \ldots, \gamma_{t}=u_{r-1}\right\}=p_{y_{t-3}, y_{t-2}, y_{t-1}, y_{t}}\left(u_{r-4}^{r-1}\right) .
$$

В результате получаем (30). 
Начальные вероятности $\alpha_{t}\left(u_{0}, \ldots, u_{t-1}\right)$ при $t=1, \ldots, r$ имеют вид

$$
\begin{aligned}
& \alpha_{1}\left(u_{0}\right)=q_{1,0, u_{0}} \varphi_{1}\left(u_{0}, y_{1}\right), \\
& \alpha_{t}\left(u_{0}, \ldots, u_{t-1}\right)=\alpha_{t-1}\left(u_{1}, \ldots, u_{t-1}\right) q_{t, u_{t-2}, u_{t-1}} \varphi_{t}\left(u_{0}^{t-1}, y_{0}^{t-1}\right), 2 \leqslant t \leqslant r .
\end{aligned}
$$

Следствие 6. Функиия правдоподобия $L\left(\varepsilon, \delta ; y_{1}^{T}\right)$, определяемая (29), допускает следующий алгоритм вычисления при $r \geqslant 3$ :

$$
L\left(\varepsilon, \delta ; y_{1}^{T}\right)=\sum_{u_{0}^{r-1} \in V_{r}} \alpha_{T}\left(u_{0}, \ldots, u_{r-1}\right),
$$

где вероятности $\left\{\alpha_{t}\right\}$ вычисляются рекуррентно по $t=1, \ldots, T$ согласно $(30),(31)$. Вичислительная сложность этого алгоритма имеет порядок $O\left(T 2^{r}\right)$.

Доказательство. Используя определение и введённые обозначения, получаем

$$
\begin{gathered}
L\left(\varepsilon, \delta ; y_{1}^{T}\right)=\mathbf{P}\left\{Y_{1}^{T}=y_{1}^{T}\right\}= \\
=\sum_{u_{0}^{r-1} \in V_{r}} \mathbf{P}\left\{Y_{1}^{T}=y_{1}^{T}, \gamma_{T-r+1}^{T}=u_{0}^{r-1}\right\}=\sum_{u_{0}^{r-1} \in V_{r}} \alpha_{T}\left(u_{0}, \ldots, u_{r-1}\right),
\end{gathered}
$$

что совпадает с (32). Вычислительная сложность алгоритма (30)-(32) относительно $T$ определяется вычислительной сложностью рекурсии $(30),(31)$ и имеет порядок $O\left(T 2^{r}\right)$.

Как видно из следствия 6 , вычислительная сложность растет с ростом $r$, поэтому на практике целесообразно использовать минимальное значение $r=3$.

Заметим, что при больших $t$ значения $\alpha_{t}$ могут выходить из диапазона чисел, представимых в формате с плавающей точкой, поэтому необходима процедура нормировки аналогично [16]. Для каждого $t=1, \ldots, T$ вычисляются значения $\alpha_{t}\left(u_{0}, \ldots, u_{r-1}\right), u_{0}, \ldots, u_{r-1} \in V$, и далее умножаются на нормировочный коэффициент $c_{t}=1 / \sum_{u_{0}^{r-1} \in V_{r}} \alpha_{t}\left(u_{0}, \ldots, u_{r-1}\right)$. В результате получаем, что логарифмическая функция правдоподобия $\log L\left(\varepsilon, \delta ; y_{1}^{T}\right)$ имеет вид:

$$
\log L\left(\varepsilon, \delta ; y_{1}^{T}\right)=-\sum_{t=1}^{T} \log c_{t}
$$

Разработанный алгоритм (30)-(32) вычисления функции правдоподобия $L\left(\varepsilon, \delta ; y_{1}^{T}\right)$ реализован и оттестирован в компьютерных экспериментах.

\section{6. Результаты компьютерных экспериментов}

Для иллюстрации состоятельности построенных в разделах 3-5 статистических оценок параметров для двух марковских моделей вкраплений проведены две серии компьютерных экспериментов.

В первой серии экспериментов для модели побитового вкрапления (2)(4) методом Монте-Карло вычислены среднеквадратические ошибки $v\{\hat{\varepsilon}\}=$ $1 / K \sum_{k=1}^{K}\left(\hat{\varepsilon}^{(k)}-\varepsilon\right)^{2}, v\{\hat{\delta}\}=1 / K \sum_{k=1}^{K}\left(\hat{\delta}^{(k)}-\delta\right)^{2}$ оценивания параметров модели на основе частотных статистик (16), (12) и выборочных корреляций (18) при следующих значениях параметров: $\varepsilon=0.3, \delta=0.15$, число прогонов $K=10^{3}$. На рис. 2,3 


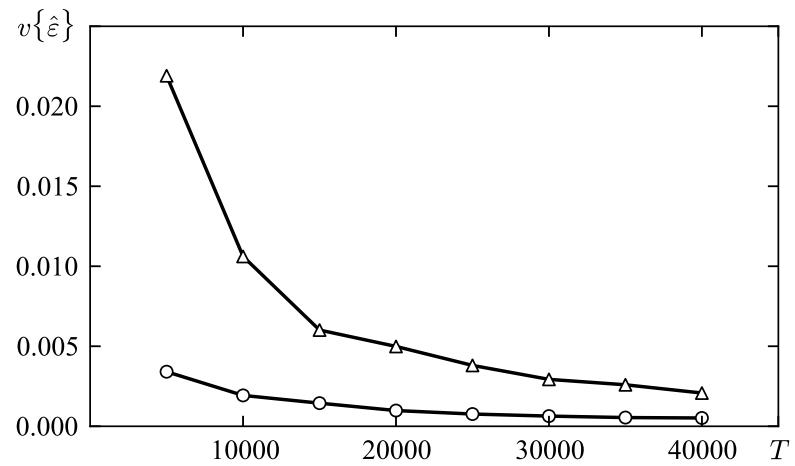

Рис. 2. Зависимость $v\{\hat{\varepsilon}\}$ от длины $T$ при $\varepsilon=0.3, \delta=0.15, K=10^{3}$

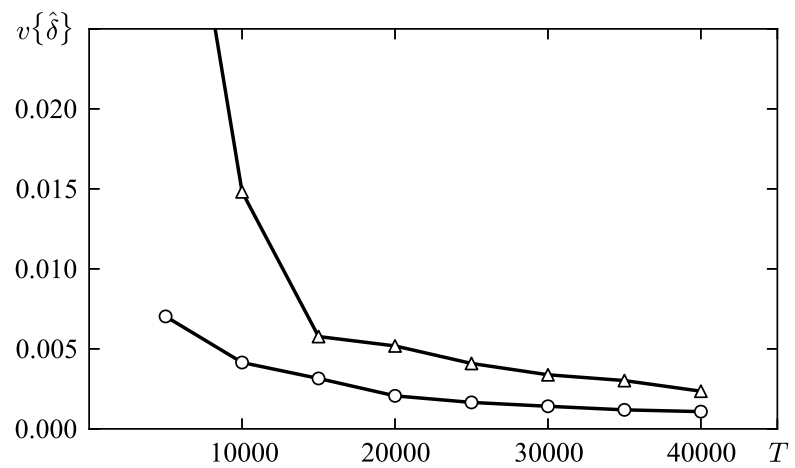

Рис. 3. Зависимость $v\{\hat{\delta}\}$ от длины $T$ при $\varepsilon=0.3, \delta=0.15, K=10^{3}$

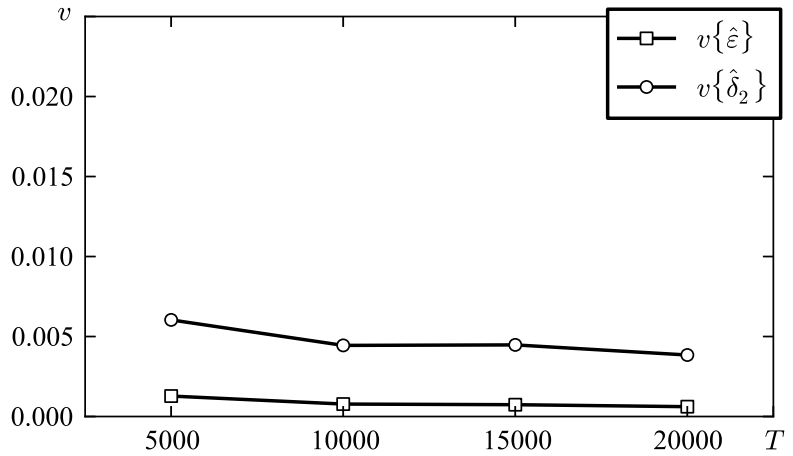

Рис. 4. Зависимость $v\{\hat{\varepsilon}\}, v\{\hat{\delta}\}$ от длины $T$ при $\varepsilon=0.3, \delta=0.3, K=10^{2}$

изображены графики зависимостей $v\{\hat{\varepsilon}\}, v\{\hat{\delta}\}$ от длины стегопоследовательности $T$. Кружками отмечены графики для частотных оценок (16), (12), треугольниками оценок на основе выборочных корреляций (18). 
Во второй серии экспериментов для модели блочного вкрапления (2)-(3),(5) вычислены среднеквадратические ошибки $v\{\hat{\varepsilon}\}, v\{\hat{\delta}\}$ МП-оценок параметров модели на основе полиномиального алгоритма (30)-(32) при следующих значениях параметров: $\varepsilon=0.3, \delta=0.3, K=10^{2}$; построение МП-оценок осуществлялось табулированием функции $L(\varepsilon, \delta)$ на решетке: $\varepsilon, \delta \in\{0.20,0.21, \ldots, 0.40\}$. На рис. 4 изображены графики зависимостей $v\{\hat{\varepsilon}\}, v\{\hat{\delta}\}$ от длины стегопоследовательности $T$.

Авторы благодарны А.М. Зубкову за вопросы и замечания, способствовавшие улучшению данной статьи.

\section{Список литературы}

1. Sullivan K., Madhow U., Chandrasekaran S., Manjunath B., Steganalysis of spread spectrum data hiding exploiting cover memory. SPIE, Electronic Imaging, Security, Steganography, and Watermarking of Multimedia Contents VII (2005) 5681, 38-46.

2. Fridrich J., Pevny T., Kodovsky J., Statistically undetectable JPEG steganography: Dead ends, challenges, and opportunities. Proc. 9-th ACM Multimedia Security Workshop (2007) $3-14$.

3. Pevny T., Bas P., Fridrich J., Steganalysis by subtractive pixel adjacency matrix. Proc. 11-th ACM Multimedia Security Workshop (2009) 75-84.

4. Shi Y. Q., Chen C., Chen W., A Markov process based approach to effective attacking JPEG steganography. Lect. Notes Comput. Sci. (2006) 4437, 249-264.

5. Харин Ю. С., Вечерко Е. В., О некоторых задачах статистической проверки гипотез в стеганографии. Вести НАН Беларуси. Сер. физ.-мат. наук (2010) 4, 5-12.

6. Kharin Yu. S., Vecherko E. V., On statistical hypotheses testing of embedding. Proc. 9-th Int. Conf. Computer Data Analysis and Modeling (2010) 2, 26-29.

7. Пономарев К. И., Параметрическая модель вкрапления и ее статистический анализ. Дискретная математика (2009) 21, №4, 148-157.

8. Bas P., Filler T., Pevny T., Break Our Steganographic System - the ins and outs of organizing BOSS. Proc. Inf. Hiding Conf. (2011).

9. Зубков А. М., Датчики псевдослучайных чисел и их применения. Сб.: Труды II Междунар. научн. конф. "Математика безопасности информационных технологий" (2003) 200-206.

10. Кемени Дж., Снелл Дж., Конечные цепи Маркова (1982).

11. Харин Ю. С., Берник В. И., Матвеев Г. В., Агиевич С. В., Математические и компъютерные основы криптологии. Новое знание, 2003.

12. Sharp T., An Implementation of Key-Based Digital Signal Steganography. Lect. Notes Comput. Sci. (2001), № 2137.

13. Ивченко Г. И., Медведев Ю. И., Математическая статистика. Высшая школа, Москва, 1984.

14. Боровков А. А., Математическая статистика. Наука, Москва, 1984.

15. Billingsley P., Statistical methods in Markov chains. Ann. Math. Statist (1961) 32, №1, 12-40.

16. Rabiner L. R., A tutorial on hidden Markov models and selected applications in speech recognition. Proc. of the IEEE (1989) 77, №2, 257-286. 